\title{
Nanoholes Permit Remarkable Light Transmission
}

\section{Passing a camel through a needle's eye--with big implications for light-emitting devices}

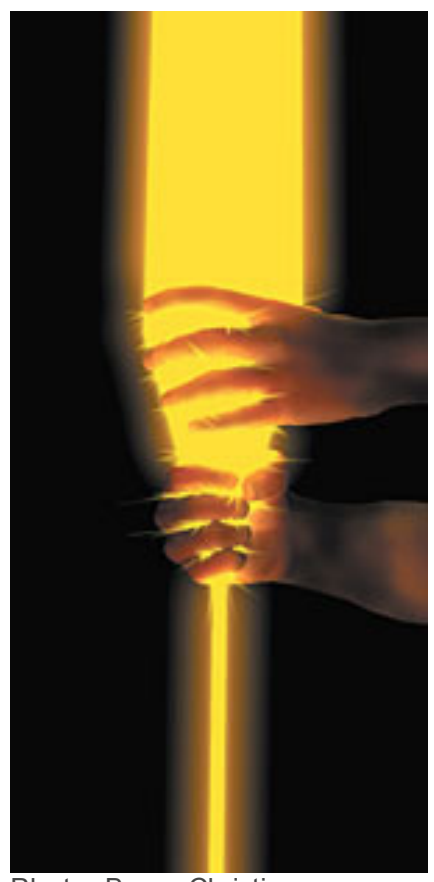

Photo: Bryan Christie

\section{BY ALEXANDER HELLEMANS // JULY 2004}

Suppose you're commanding an army of archers and launching an attack against an enemy force separated from you by a huge mesh screen. Your army's wobbling arrows all get stuck in the screen or bounce off it. But suppose something could influence the mesh of that screen so that all the arrows passed through it, even if the arrows' wobble is much greater than the size of the holes in the mesh.

Something very much like that is being accomplished in the new field of subwavelength nanostructures. Researchers are finding ways to send light through metal films perforated by holes with diameters much smaller than the wavelength of the light. The classical rules of optics say that this is impossible, but the tricks being developed, as well as a growing understanding of what makes the tricks work, could lead to more efficient solid-state lasers, LEDs, and other electronic components.

A Japanese group, for example, is developing a new solid-state surface-emitting laser made from a material with a very high index of refraction. The material reflects the light generated internally so well that light emission outside the device is poor. The problem could be solved by placing a porous metallic film at the interface between the high-index material and the external world. The film would have certain properties that the field of subwavelength nanostructures is devoted to exploring.

The basic mechanism permitting light to pass through holes that are too small for it depends on plasmons, tiny electron waves set up in the surface of the metal film when it is struck by the light. Researchers at the Louis Pasteur University in Strasbourg, France, and the Tokyo Institute of Technology have succeeded in obtaining images of the plasmons formed around a subwavelength nanohole. That work shows that plasmons set up tiny magnetic dipoles around the rims of the holes. Other researchers have shown that the shape of a nanohole and the polarization of the incident light affect the light transmission, too.

The line of investigation that culminated in the most recent advances began in 1998 when Thomas Ebbesen at Louis Pasteur University discovered, to his surprise, that light passed through arrays of holes in a metal film that had diameters about 10 to 20 percent the wavelength of the light. The silver and gold films used in these experiments are typically a few hundred nanometers thick, and the holes, about $150 \mathrm{~nm}$ in diameter, are made by electron-beam or ion-beam milling. Ebbesen reported that light did not just pass through the holes but seemed to be squeezed through them: twice as much light passed through as would normally be allowed by the area of the holes occupying the film. The phenomenon became known as "extraordinary transmission" because classical optics predicts that light cannot pass through a hole smaller than its wavelength.

The extraordinary transmission became even more extraordinary when Ebbesen and his team reported in 2002 that a single subwavelength hole can transmit even more light than expected when it is surrounded with concentric circular grooves. "You can get transmissions of 20 times the whole area. The corrugation acts like an antenna and pushes the light through," said Ebbesen. He reported further that such a "bull's-eye" can focus light, like a lens. Applications have been demonstrated already. For example, a Japanese team at the Tokyo Institute of Technology, having mounted such a bull's-eye structure on top of a vertical-cavity surface-emitting laser (VCSEL), reported last year that they were able to increase the transmitted light intensity considerably.

Data storage is another application. There, the bull's-eye would be able to produce a subwavelength bright spot on an optical recording medium. 
Now, in a paper just accepted by Optics Letters, Ebbesen reports how his team succeeded in photographing light coming from the plasmons around a subwavelength aperture. The images confirm that the light striking the metallic film sets up plasmons that create tiny magnetic dipoles around the holes that line up with the polarization of the incoming light. Polarization effects, which could be exploited to engineer materials for maximum transmissivity, also have been observed by a group at the University of Victoria in Canada and by a Dutch-French team at the University of Twente in Enschede, Netherlands, the Institute for Atomic and Molecular Physics in Amsterdam, and the Fresnel Institute in Marseilles, France. This team, led by L. ("Kobus") Kuipers at Twente, observed that light is transmitted when the polarization plane of the light is perpendicular to the longer side of a rectangular hole. The Canadian team observed a polarization effect in elliptical holes.

It has been determined that the shape, in addition to the size, of a subwavelength aperture can play an important role in the transmission of light. The Kuipers team has observed that more light passes through rectangular 75-by-225$\mathrm{nm}$ subwavelength apertures than through round apertures of 190-nm diameter, even though the round apertures are bigger. Kuipers believes this result shows that electromagnetic-wave resonances are appearing in the rectangular--but not the round--holes and that these resonances also influence the transmission of light.

Roy Sambles, who experiments with the transmission of microwaves through subwavelength apertures at the University of Exeter in the United Kingdom, believes that the rectangular shapes are acting as waveguides. That might open a second avenue for enhancing the light-emitting properties of devices of all sorts. 